\title{
Fluctuation-induced order in two-dimensional colloidal clusters
}

\author{
R. Bubeck, P. Leiderer and C. Bechinger \\ Department of Physics, University of Konstanz - D-78457 Konstanz, Germany
}

(received 26 April 2002; accepted in final form 21 August 2002)

PACS. 82.70.Dd - Colloids.

PACS. 64.60. Cn - Order-disorder transformations; statistical mechanics of model systems.

PACS. 83.10.Tv - Structural and phase changes.

\begin{abstract}
The behavior of a finite number $(N=29)$ of paramagnetic colloidal particles in $2 \mathrm{D}$ circular hard-wall cavities is investigated. By applying a magnetic field $B$, the interaction between the particles is varied. We demonstrate that the angular diffusion of the particles which are arranged in shells is highly anisotropic and shows a non-monotonic behavior as a function of $B$. When reducing radial particle fluctuations in one shell by a ring-shaped optical tweezer, the relative angular diffusion between adjacent shells increases again. This clearly demonstrates that radial particle fluctuations are responsible for an enhanced registration between adjacent shells.
\end{abstract}

Thermal fluctuations are well known to have a strong influence on the phase behavior of two-dimensional (2D) systems compared to their three-dimensional counterparts [1]. The most striking evidence for such a difference is probably the melting transition which is, in 2D, predicted to occur via two sequential defect-driven continuous phase transitions as described by the KTHNY theory (see, e.g., [2]). Amongst conveniently accessible experimental systems for basic investigations of 2D melting colloidal particles have been established as model systems because they allow direct optical observation of topological defects and comparison to theoretical predictions [3-5]. However, while the KTHNY theory applies only for extended $2 \mathrm{D}$ systems, much less (both experimentally and theoretically) is known about the properties of $2 \mathrm{D}$ systems which are only comprised of a few particles (typically less than $N=100$ ). Due to the finitness of such systems a different melting scenario compared to infinite systems is expected which may be important for the understanding of melting and freezing of, e.g., small $2 \mathrm{D}$ clusters or ions in radiofrequency traps [6]. It has been experimentally demonstrated that when super paramagnetic colloidal particles are confined to a circular hard-wall cavity, the particles at low effective temperatures $T_{\text {eff }}$ do not crystallize in a triangular lattice, but are rather arranged in a shell-like structure with both positional and orientational order. Upon increasing the temperature first orientational order between adjacent shells is lost which is typically refered to as intershell rotation. As the temperature is increased further, a "re-entrant" ordered phase is observed which is followed by complete melting of the cluster [7]. While intershell rotation is also observed in numerical studies of finite $2 \mathrm{D}$ systems of ions or electrons in circular cavities, the "re-entrant" ordered phase as found in the experiments, is still 
controversially discussed by several groups $[8,9]$. In particular, it is not clear to what amount radial particle fluctuations may lead to a non-monotonic behavior of the angular-diffusion coefficient as this was recently suggested [10].

In this paper we present an experimental study of the behavior of a $2 \mathrm{D}$ system with $N=29$ particles interacting via a dipole potential, which are confined to a circular hard-wall cavity. To study the role of radial particle fluctuations in detail, we additionally superimposed a ring-shaped optical laser tweezer to the second shell which enhances the radial localization of the particles therein. As a result, we observe that the angular motion between adjacent shells considerably increases. This strongly supports the important role of radial fluctuations for the angular registration and orientational order in such systems.

The experimental setup is similar to that used in previous experiments, therefore it will be described here only in brief $[7,11]$. We used aqueous suspensions of super paramagnetic colloidal particles with a diameter of $4.55 \mu \mathrm{m}$ which have been purchased from Dynal (DynaBeads $4.55 \mu \mathrm{m}$, Dynal, Lot-No. B20100) and stabilized them with $2.0 \mathrm{mg} / \mathrm{ml}$ SDS (sodium dodecyl sulfate). As substrates for the colloidal system we used fused silica plates which were coated with a $3-4 \mu \mathrm{m}$ thick smooth film of poly (methyl methacrylate) (PMMA) by spin casting. This coating is necessary to achieve a high mobility of the particles and prevent them from sticking to the surface. After the PMMA deposition, the substrate was heated to about $150^{\circ} \mathrm{C}$ and a copper grid with circular openings of $72 \mu \mathrm{m}$ in diameter and a thickness of $15 \mu \mathrm{m}$, as is typically used as sample holder in transmission electron microscopy, was pressed onto the soft PMMA film. This procedure yields typically several tens of circular compartments (diameter $72 \mu \mathrm{m}$ ) with perpendicular hard walls. Because the copper grids are not magnetic, the particle-wall interaction can be regarded to good approximation as hard-wall-like. When colloidal suspension is added onto the copper grid, the particles start to sediment towards the patterned bottom plate. Due to the high density of the particles $\left(\rho=1.7 \mathrm{~g} / \mathrm{cm}^{3}\right)$, they are confined by gravity to a $2 \mathrm{D}$ system in each compartment. In our experiments the number density in different compartments varied statistically between about 25 and 45 , depending on the particle concentration in the suspension. A second silica plate was assembled on top of the bottom plate with a $1 \mathrm{~mm}$ O-ring as a spacer to avoid contamination of the suspension with dust. In the presence of an external magnetic field $B$, which was applied perpendicular to the sample plane, a magnetic moment $M(B)$ is induced in the particles, which leads to a repulsive magnetic-dipole pair potential $V_{i, j}=\mu_{0} M^{2}(B) / 4 \pi r_{i j}^{3}$, where $r_{i j}$ denotes the distance between particles $i$ and $j$.

The colloidal spheres were imaged with a home-built inverted video microscope onto a CCD-camera. An image-processing system is used to determine time-resolved particle trajectories by means of a particle recognition algorithm. From this we obtained the particle mean-square displacements (MSD). In order to modify the extent of radial particle fluctuations, a laser beam (HeNe, $\lambda=633 \mathrm{~nm}$ ) was deflected from a 2D computer-controlled galvanostatic driven mirror and then directed from the top into the sample cell. This setup served as a scanning optical tweezer for the particles [12]. The shape and size of the laser trap could be easily adjusted by the computer. The repetition rate of the tweezer pattern is about $400 \mathrm{~Hz}$, i.e. much faster than the diffusive motion of the particles on a homogeneous substrate $\left(D=0.035 \mu \mathrm{m}^{2} / \mathrm{s}[13]\right)$. We did not observe that the scanning direction of the laser tweezer introduced any asymmetries with respect to the angular particle motion. Therefore, the laser tweezer can be considered to provide a static potential to the particles [14].

The effective interaction between the paramagnetic spheres can be conveniently characterized by means of a dimensionless plasma parameter $\Gamma=q^{2} /\left(a^{3} k_{\mathrm{B}} T\right)$ with $q^{2}=\mu_{0} M^{2}(B) / 4 \pi$ and $a=2 R / N^{1 / 2}$ the mean interparticle distance [10]. Here $R$ corresponds to the radius of the circular hard-wall confinement, $k_{\mathrm{B}}$ to the Boltzman constant, and $T$ to the temperature 


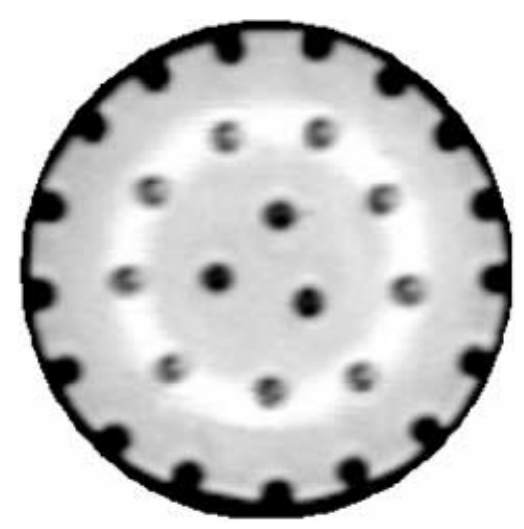

Fig. 1 - Photograph of a circular hard-wall cavity which is occupied by a $2 \mathrm{D}$ colloidal system of 29 particles. The concentric bright ring corresponds to a scanned laser beam which acts as an optical tweezer for the particles in the second shell.

of the suspension. Accordingly, $\Gamma$ is proportional to the inverse effective temperature of the system. Within the range of magnetic fields used in this work, $M$ is in good approximation proportional to $B$. (Please note that the above definition of $\Gamma$ leads to plasma parameters which are for $N=29$ a factor of 2.2447 smaller than those in ref. [7]). In our experiments we varied $\Gamma$ by changing $B$ and kept the temperature fixed at $T=295 \pm 1 \mathrm{~K}$. For each magnetic field $B$, we measured the particle trajectories with a lateral and temporal resolution of $0.25 \mu \mathrm{m}$ and $20 \mathrm{~ms}$, respectively. In contrast to previous measurements, where trajectories are followed only for 30 minutes [7], here we increased the measuring time up to several hours.

Figure 1 shows a real space image of $N=29$ particles in a hard-wall confinement for $\Gamma=67.3$ corresponding to the highest value in this work. It can be seen that the particles are arranged in three shells with 3, 9, and 17 particles, respectively. This configuration has been shown to correspond to the ground state of such a system [7]. The bright ring which is visible at the position of the second shell corresponds to the laser tweezer which is scanned in a ring-shaped manner in the sample plane. Special care was taken that the center of the laser ring corresponded to the center of the circular cavity and that the position of highest intensity of the Gaussian-shaped laser beam was adjusted to the center of mass of the particles in the second shell. Accordingly, the laser tweezer provides an optical trap for the particles in the second shell and reduces their radial fluctuations to an amount which is determined by the intensity of the laser [15]. As can be seen from the radial particle density which is plotted in fig. 2, without and with the laser tweezer turned on as open and closed symbols, respectively, a laser intensity of $1.85 \mathrm{~mW}$ reduces the half-width of the radial extent of the particles in the second shell by about $20 \%$. In contrast, the radial distribution of the inmost and outmost shells are essentially unchanged. This clearly demonstrates that the laser tweezer is only affecting the particles in the second shell.

Besides gradient forces which reduce lateral particle fluctuations, the laser beam is also exerting a vertical pressure onto the colloidal spheres. To determine the extent of this force, which could also change the diffusion coefficient, we employed total internal reflection microscopy (TIRM) $[16,17]$ because this method allows force measurements with a resolution of $10 \mathrm{fN}$ [18]. TIRM utilizes an evanescent field, which is formed upon total reflection of a weak laser beam at a solid-liquid interface. When a colloidal particle, which is suspended in the liquid, approaches the interface, it is illuminated by the evanescent field and scatters 


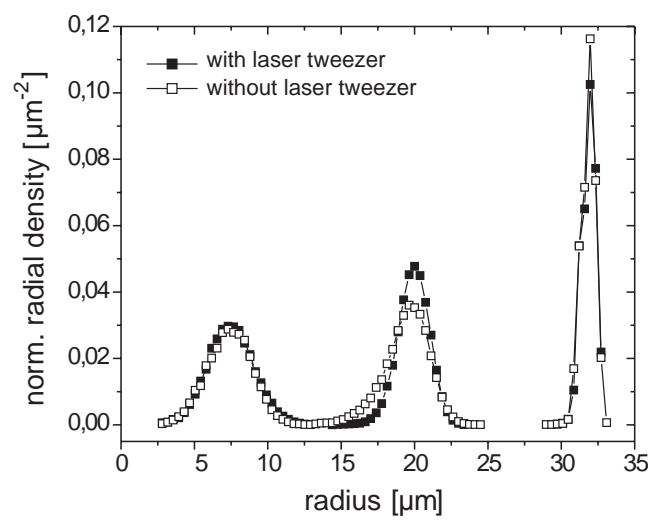

Fig. 2 - Effect of the optical tweezer on the radial density of the particle distribution for $\Gamma=15$. The open and closed symbols correspond to the values without and with the tweezer, respectively. While the radial particle fluctuations of the second shells are influenced by the tweezer, no effect is observed in the other shells.

light. From the intensity distribution, the particle-wall interaction potential can be calculated. This potential is typically given by superposition of electrostatic, gravitational, and -in case of a laser tweezer - light forces. To study the effect of light forces in our experiments, we directed the HeNe beam (with the galvanostatic drive turned off) from below (!) onto a particle. Afterwards the laser intensity was increased until the light force compensated the well-known gravitational force of the colloidal sphere. This yields an estimate for the vertical light force which is exerted onto a particle. For the above-mentioned ring-shaped HeNe tweezer with $1.85 \mathrm{~mW}$, we obtain a light force directed towards the substrate corresponding to only about $2.5 \%$ of the gravitational force of the superparamagnetic spheres. Therefore, in our experiments vertical light forces can be neglected to a good approximation.

In order to characterize the angular diffusion of the particles in the inmost shell relative to the angular motion of the second shell in a quantitative manner, we first transformed the particle's trajectories into a reference frame which follows the collective angular motion of the second shell. As a criterion to determine to which shell a particle is attributed, we used the position of the minima in the radial particle distribution (see fig. 2). As can be seen, the radial density in the minima decays almost to zero, which demonstrates that particle jumps between adjacent shells even at relatively small $\Gamma$ are very rare.

From the transformed particle trajectories (we only used those parts of the trajectories where - according to the above shell definition - no shell jumps occurred), we obtained the time-dependent relative angular mean-square displacement $\left\langle\Delta \Theta^{2}(t)\right\rangle=\left\langle\left(\theta\left(t_{0}+t\right)-\right.\right.$ $\left.\left.\theta\left(t_{0}\right)\right)^{2}\right\rangle_{t_{0}, N_{1}}$ of the particles in the inmost shell $\left(N_{1}\right)$, where the brackets correspond to time and particle averaging. The corresponding data without applying the ring-shaped laser trap are plotted in fig. 3 for $\Gamma=12.5$ as open symbols. After about $100 \mathrm{~s}$ the transition from the short to the long-time diffusion behavior occurs. From the slope of the linear increase at longer times the angular-diffusion coefficient $D_{\Theta}=\lim _{t \rightarrow \infty}\left\langle\Delta \Theta^{2}(t)\right\rangle / 2 t$ can be calculated. Figure 4 shows the result of $D_{\Theta}$ for $8.7 \leq \Gamma \leq 67.3$ as open symbols. With decreasing plasma parameters down to $\Gamma \approx 15$ the angular diffusion of the first shell increases, which is the signature of the well-known intershell diffusion [10]. Below $\Gamma=15$, however, $D_{\Theta}$ decreases until the shell structure for $\Gamma<8.7$ finally smears out due to intershell diffusion. In this regime the angular-diffusion coefficient is not defined any longer. Such a non-monotonic behavior is 


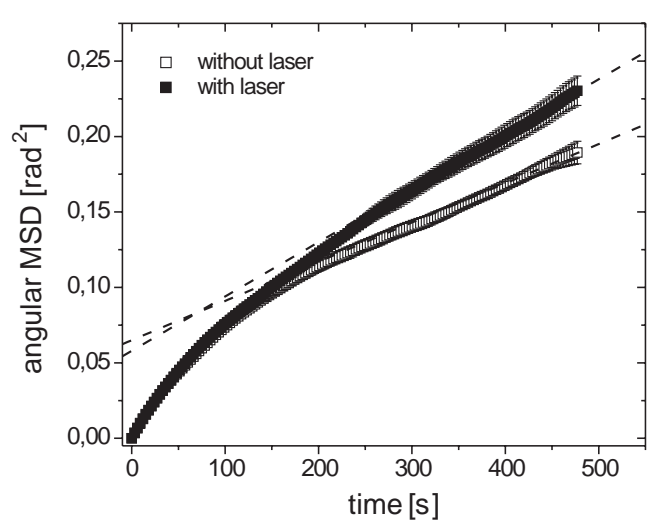

Fig. 3 - Time-dependent angular mean-square displacements (AMSD) for $\Gamma=12.5$ without (open symbols) and with (closed symbols) a ring-scanned optical tweezer. Towards long-time scales, the curves can be fitted to straight lines (dashed) whose slope is proportional to the angular-diffusion coefficient.

consistent with both our previous results [7] as well as the BD-simulations of Schweigert et al. [10] and has been interpreted to be a result of radial particle fluctuations which stabilize the orientational order between adjacent shells.

To investigate the role of radial particle fluctuations in detail, we superimposed a laser tweezer to the second shell as described before and repeated the above measurements. The closed symbols in fig. 3 correspond to $\left\langle\Delta \Theta^{2}(t)\right\rangle$ for $\Gamma=12.5$ in the presence of the laser tweezer. As can be seen, the linear slope at longer time scales is considerably larger than in the absence of the tweezer, indicating that the angular-diffusion coefficient with the optical trap $D_{\Theta}^{\mathrm{L}}$ is larger than $D_{\Theta}[19]$. Figure 4 shows a direct comparison between $D_{\Theta}^{\mathrm{L}}$ (closed symbols) and $D_{\Theta}$ (open symbols) as a function of $\Gamma$. The accessible range during the tweezer experiments was limited to $12.5 \leq \Gamma \leq 26$. This is due to the fact that above $\Gamma=26$ the form of the second shell is slightly distorted into a triangular shape because of the threefold

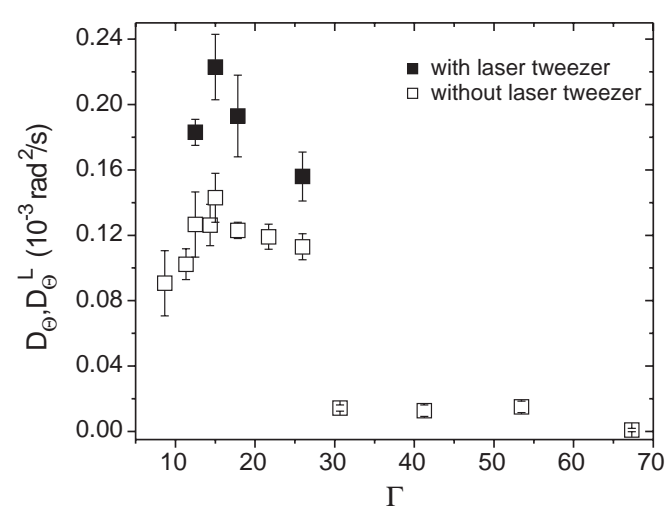

Fig. 4 - Comparison of the angular-diffusion coefficients without $D_{\Theta}$ (open symbols) and with $D_{\Theta}^{\mathrm{L}}$ (closed symbols) a ring-shaped laser tweezer. As can be seen, the diffusion coefficient is enhanced by the laser tweezer which demonstrates the role of radial particle fluctuations for the stabilization of the angular order. 
symmetry of the inmost shell [7]. Below $\Gamma=12.5$, the particle-particle interaction becomes already so weak that the presence of the laser tweezer is changing the particle configuration, i.e. by attracting particles from the inmost into the second shell. As can be seen, $D_{\Theta}^{\mathrm{L}}$ is over the entire range of investigated $\Gamma$-values always considerably larger than $D_{\Theta}$ which demonstrates that a partial suppression of radial particle fluctuations enhances the angular diffusion between adjacent shells. This behavior becomes immediately understandable in terms of the abovementioned model where radial particle fluctuations increase the angular registration between shells. Accordingly, if these fluctuations are reduced, as this is done by the laser tweezer, the registration is weakened which leads then to an increased angular-diffusion coefficient. Since this is in very good agreement with fig. 4, our data strongly support the important role of radial particle fluctuations for the melting and freezing in such systems.

While the reduction of the radial fluctuations induced by the scanned optical tweezer as discussed here is relatively small, for sufficiently strong radial constraints in principle a monotonic behavior of the angular-diffusion coefficient is expected. This is also confirmed by very recent Monte Carlo simulations [20]. Experimentally, strong radial constraints are difficult to achieve because the particle shell which is, in the absence of the laser tweezer, slightly facetted will be distorted by the presence of a strong light field. In addition, a strong optical tweezer can also affect adjacent shells due to light scattering.

It is important to note that the behavior observed here strongly depends on the detailed shape of the confining cavity. As has been pointed out earlier, a non-monotonic behavior of $D_{\Theta}$ as a function of the plasma parameter is only observed if the expansion of the system as a function of $\Gamma$ is essentially zero $[7,10]$. While this is fulfilled for hard-wall cavities, parabolic walls can lead to a substantial expansion of the system. As a result, the mean distance between adjacent shells increases with increasing $\Gamma$ and thus counteracts the mechanism described above. As a result, a non-monotonic behavior in $D_{\Theta}$ is absent in parabolic confinements [10,21].

In summary, we have studied the melting process of a $2 \mathrm{D}$ colloidal cluster which is confined to a circular hard-wall cavity. Our results support earlier observations that the angular mobility of adjacent shells shows a non-monotonic behavior which has been suggested to be explained in terms of radial particle fluctuations which lead to an enhanced angular registration of adjacent shells at low plasma parameters. This model has been experimentally confirmed by reducing radial particle fluctuations by means of a ring-shaped laser trap which was superimposed to the second shell. As expected, a reduction of radial fluctuations leads to a decoupling of adjacent shells and thus to a high angular mobility.

We gratefully acknowledge the help of L. HELDEN for the TIRM measurements and financial support from the Deutsche Forschungsgemeinschaft (SFB 513).

\section{REFERENCES}

[1] Lindemann F. A., Phys. Zeits., 11 (1910) 609.

[2] Nelson D. R., Phase Transtions and Critical Phenomena (Academic Press, London) 1983.

[3] Murray C. A. and Winkle D. H. V., Phys. Rev. Lett., 58 (1987) 1200.

[4] Marcus A. H. and Rice S. A., Phys. Rev. E, 55 (1997) 637.

[5] Zahn K., Lenke R. and Maret G., Phys. Rev. Lett., 82 (1999) 2721.

[6] Birkl G., Kassner S. and Walther H., Europhys. News, 23 (1992) 143.

[7] Bubeck R., Bechinger C., Neser S. and Leiderer P., Phys. Rev. Lett., 82 (1999) 3364.

[8] Schweigert I. V., Schweigert V. A. and Peeters F. M., Phys. Rev. Lett., 86 (2001) 4712. 
[9] Rinn B. and Maass P., Phys. Rev. Lett., 86 (2001) 4711.

[10] Schweigert I. V., Schweigert V. A. and Peeters F. M., Phys. Rev. Lett., 84 (2000) 4381.

[11] Bubeck R., Neser S., Bechinger C. and Leiderer P., Progr. Colloid Polym. Sci., 110 (1998) 41.

[12] Ashkin A., Biophys. J., 61 (1992) 569.

[13] Due to an typographical error the diffusion coefficient in a previous paper was falsely reported. Instead of $0.35 \mu \mathrm{m}^{2} / \mathrm{s}$ the correct value is $0.035 \mu \mathrm{m}^{2} / \mathrm{s}$.

[14] Faucheux L. P., Stolovitzky G. and Libchaber A., Phys. Rev. E, 51 (1995) 5239.

[15] Ashrin A., Phys. Rev. Lett., 24 (1970) 156.

[16] Prieve D. C., Adv. Colloid Interface Sci., 82 (1999) 93.

[17] Walz J. Y., Current Opin. Colloid Interfaces Sci., 2 (1997) 600.

[18] Rudhardt D., Bechinger C. and Leiderer P., J. Phys. Condens. Matter, 11 (1999) 10073.

[19] Because the short-time behavior is almost identical compared to that without laser tweezer, this demonstrates that the short-time diffusional behavior is not affected by the presence of the laser tweezer.

[20] Henseler P. and Nielaba P., unpublished results.

[21] Bubeck R., Leiderer P. and Bechinger C., Progr. Colloid Polym. Sci., 118 (2001) 73. 